\title{
Fatal Neutropenic Colitis and Clostridium Septicum Bacteremia in a Breast Cancer Patient
}

\section{Michal Holub', David Řezáč', Martina Čurdová ${ }^{2}$}

${ }^{1}$ Department of Infectious Diseases, First Faculty of Medicine, Charles University and Military University Hospital Prague, Prague, Czech Republic;

${ }^{2}$ Department of Clinical Microbiology, Military University Hospital Prague, Prague, Czech Republic

Received June 2, 2021; Accepted August 3, 2021.

Key words: Febrile neutropenia - Colitis - Sepsis - Clostridium septicum

Abstract: A fatal case of 67-year-old female with metastatic breast cancer on chemotherapy complicated with febrile neutropenia, colitis and sepsis due to Clostridium septicum is presented. Important clinical symptoms, laboratory and radiology findings together with therapy and outcome of neuropenic colitis are also discussed.

This study was supported by the project M01012 of the Ministry of Defence of the Czech Republic.

Mailing Address: Prof. Michal Holub, MD., PhD., Department of Infectious Diseases, First Faculty of Medicine, Charles University and Military University Hospital Prague, U Vojenské nemocnice 1200, 16902 Prague 6, Czech Republic; Phone: +420 973208 576; e-mail: michal.holub@uvn.cz 


\section{Introduction}

Febrile neutropenia (FN) is serious complication of chemotherapy in cancer patients with solid tumours. As medical emergency FN must be swiftly managed including immediate administration of broad-spectrum bactericidal antibiotics with piperacillin/tazobactam being the most frequently used for the community-acquired and meropenem with vancomycin for hospital-acquired infections. Also, extensive work-up is an integral part of initial approach to a patient with FN. This consist of obtaining numerous blood cultures and specimens from different body sites as well as the use of imaging methods that may help to localize the focus of infection. The results of cultivation are obtained with a delay of one to two days; however, the detection of infectious focus with imaging methods is rapid having impact on choice of empirical antibiotic therapy, source control and assessment of the outcome.

\section{Case report}

A 67-year-old female presented to the emergency department three hours after acute onset of fever, chills, nausea, vomiting, lower abdominal pain, and bowel and
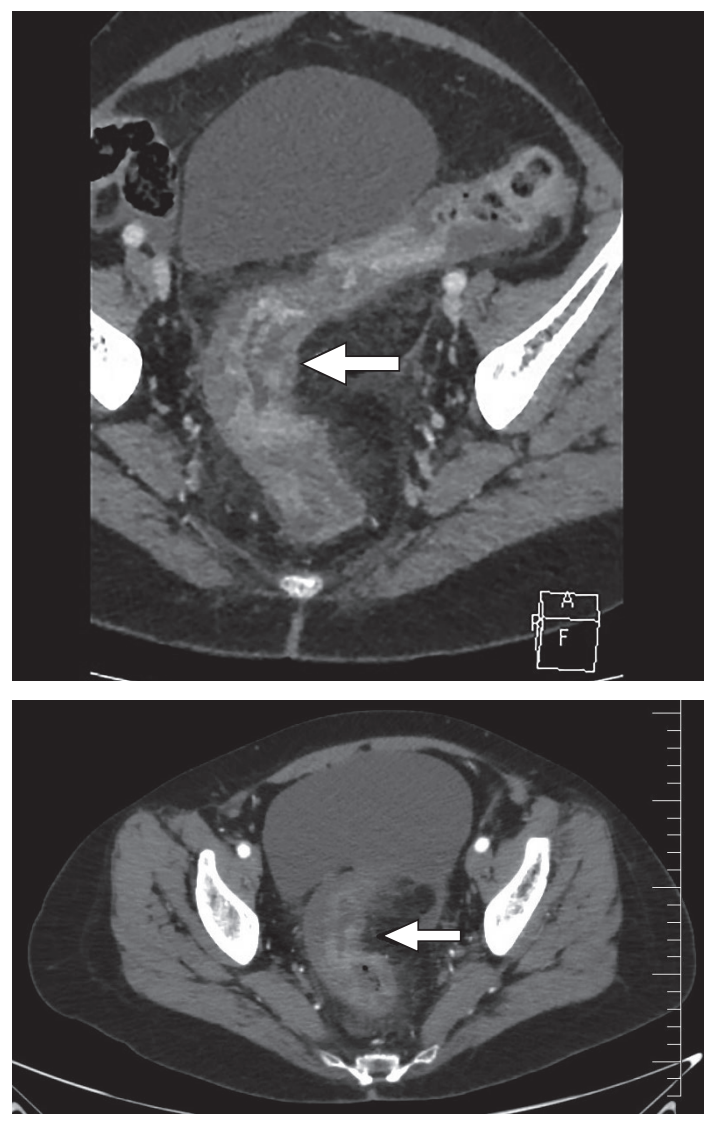

Figure $1-a, b)$ Computed tomography scans of lower abdomen indicating sigmoid walls thickening. 


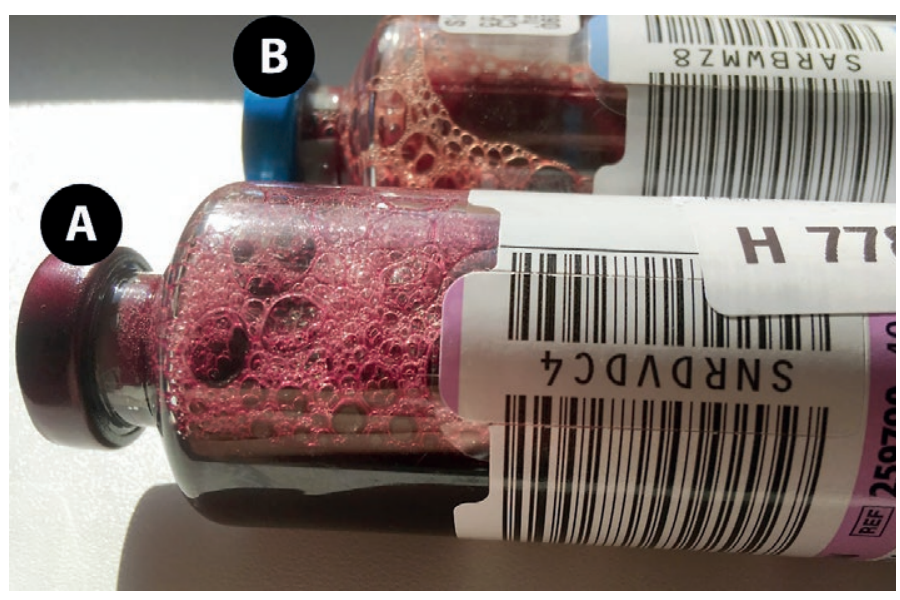

Figure 1 - c) Anaerobic blood culture bottle $A$ with gas and colour of port wine and aerobic blood culture bottle $B$ without bacterial growth; d) Gram stain of positive blood culture (arrows indicate Gram-positive rods).

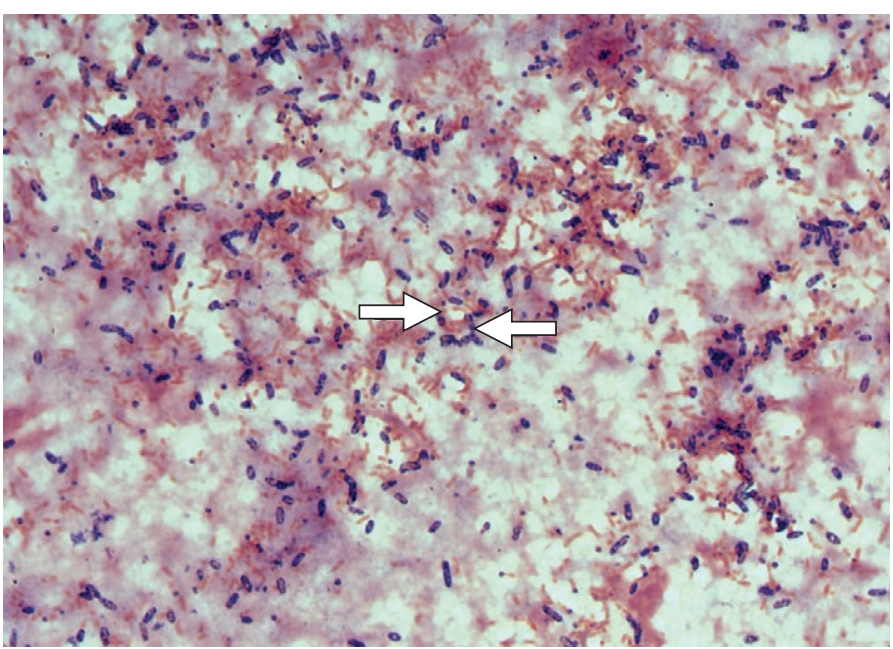

urinary urgency. The patient had recently finished the first cycle of neoadjuvant chemotherapy with standard-dose epirubicin and cyclophosphamide for metastatic breast cancer. On examination, she was febrile with abdominal distension and suprapubic tenderness. Laboratory tests revealed a low hemoglobin level (108 g/l), leukopenia $\left(0.71 \times 10^{9} / \mathrm{I}\right)$, neutropenia $\left(0.13 \times 10^{9} / \mathrm{I}\right)$ and an elevated procalcitonin level $(1.260 \mathrm{ng} / \mathrm{ml})$. An underlying diagnosis of $\mathrm{FN}$ was established. Because of suspicion for acute abdomen, abdominal computed tomography was performed immediately with finding of colitis of the sigmoid colon to the rectum with suspicion of a developing abscess in the transition from the sigmoid colon to the rectum (Figure $1 \mathrm{a}$ and $\mathrm{b}$ ). The patient received empirical antibiotic therapy with meropenem and was transferred to the intensive care unit where she died from septic shock two days later before surgery was possible. At that time, the anaerobic blood 
culture demonstrated bacterial growth with gas production and hemolysis (Figure 1c) and Gram stain of the pre-cultivated blood from the blood culture bottle revealed numerous Gram-positive rods with a terminal spores and drumstick-like shape (Figure 1d). Clostridium septicum was identified from the blood culture and the diagnosis of clostridial bacteremia was established.

\section{Discussion}

Clostridium septicum is a highly virulent pathogen which is associated with colorectal malignancy, hematological malignancy, immunosuppression, diabetes mellitus and cyclical neutropenia. Clinical presentation of the infection may include sepsis, gas gangrene, and mycotic aortic aneurysms (Jessamy et al., 2016). Also, it is well known that clostridial bacteremia is associated with substantial mortality reaching $20 \%$ in cancer patients and C. septicum is the second most common cause of clostridial bacteremia in patients with solid tumours (Hammond et al., 2014). Nausea, vomiting, abdominal pain, hypotension, acute hemolysis and focal gastrointestinal signs are associated with increased 7-day mortality. Importantly, neutropenic colitis should always be considered in cancer patients receiving chemotherapy with $\mathrm{FN}$ and abdominal pain, because these complications represent a life-threatening condition requiring aggressive therapy including intravenous antibiotics and surgical intervention (Rodrigues et al., 2017). For antibiotic selection, usual anaerobic coverage can include penicillins, metronidazole or carbapenems. However, if clostridium has been identified, combination with clindamycin is preferred because it may block production of $C$. septicum toxin - the main virulent factor of the pathogen responsible for intravascular hemolysis and tissue necrosis (Stevens et al., 2014). It is worth noting that neutropenic colitis can be due to a variety of bacterial species with Bacteroides and Clostridium species being the most frequently isolated; however, when $C$. septicum is the cause of neutropenic colitis, sepsis and with a fulminant course and death commonly occur (Nesher and Rolston, 2013).

\section{References}

Hammond, S. P., Buckley, M. W., Petruzziello, G., Koo, S., Marty, F. M., Baden, L. R. (2014) Clinical characteristics and outcomes of clostridial bacteremia in cancer patients. Clin. Microbiol. Infect. 20(8), 752-757.

Jessamy, K., Ojevwe, F. O., Ubagharaji, E., Sharma, A., Anozie, O., Gilman, C. A., Rawlins, S. (2016) Clostridium septicum: An unusual link to a lower gastrointestinal bleed. Case Rep. Gastroenterol. 10(2), 489-493.

Nesher, L., Rolston, K. V. (2013) Neutropenic enterocolitis, a growing concern in the era of widespread use of aggressive chemotherapy. Clin. Infect. Dis. 56(5), 711-717.

Rodrigues, F. G., Dasilva, G., Wexner, S. D. (2017) Neutropenic enterocolitis. World J. Gastroenterol. 23(1), $42-47$.

Stevens, D. L., Bisno, A. L., Chambers, H. F., Dellinger, E. P., Goldstein, E. J., Gorbach, S. L., Hirschmann, J. V., Kaplan, S. L., Montoya, J. G., Wade, J. C. (2014) Practice guidelines for the diagnosis and management of skin and soft tissue infections: 2014 update by the Infectious Diseases Society of America. Clin. Infect. Dis. 59(2), e10-e52. 